Enfoques. Revista de Investigación en Ciencias de la Administración http://doi.org/10.33996/revistaenfoques.v2i7.39 No. 7 | Volumen 2 | Julio - Septiembre 2018 http://revistaenfoques.org ISSN: $2616-8219$

\section{LA APLICACIÓN DE PAUSAS ACTIVAS COMO ESTRATEGIA PREVENTIVA DE LA FATIGA Y EL MAL DESEMPEÑO LABORAL POR CONDICIONES DISERGONÓMICAS EN ACTIVIDADES ADMINISTRATIVAS}

\author{
THE APPLICATION OF ACTIVE BREAKS AS A PREVENTIVE \\ STRATEGY FOR FATIGUE AND POOR WORK PERFORMANCE DUE \\ TO DYSERGONOMIC CONDITIONS IN ADMINISTRATIVE \\ ACTIVITIES
}

Carlos Jaspe, Fernando López y Soleini Moya
Artículo recibido 22 abril 2018 | Arbitrado mayo-junio 2018 | Publicado 01 de julio 2018

\section{Resumen}

Artículo que expresa los aspectos más resaltantes de analizar la aplicación de pausas activas como estrategia preventiva de la fatiga y el mal desempeño laboral por condiciones disergonómicas en actividades administrativas de una empresa manufacturera ubicada en el municipio Valencia, estado Carabobo, con el propósito de prevenir enfermedades ocupacionales, identificando condiciones disergonómicas y generar bienestar en los trabajadores en pro del desempeño laboral. Metodológicamente se trató de una investigación de campo descriptivo, se utilizó como técnicas de recolección de datos, la observación directa y la encuesta, en conjunto con los instrumentos: guía de observación y cuestionario los cuales fueron aplicados a una muestra de (21) trabajadores del área administrativa. Como resultado se recogió una alternativa para aumentar el desempeño laboral, disminuir dolencias físicas, estrés, desórdenes emocionales y riesgos laborales asociados a la actividad diaria.

Palabras clave: Pausas activas; desempeño laboral; condiciones disergonómicas

\begin{abstract}
Article that expresses the most important aspects of analyzing the application of active breaks as a preventive strategy of fatigue and poor work performance due to dysergonomic conditions in administrative activities of a manufacturing company located in the municipality of Valencia, Carabobo state, with the purpose of preventing diseases occupational, identifying dysergonomic conditions and generate well-being for workers in favor of job performance. Methodologically it was a descriptive field investigation, it was used as data collection techniques, direct observation and the survey, together with the instruments: observation guide and questionnaire which were applied to a sample of (21) workers of the administrative area. As a result, an alternative was collected to increase work performance, reduce physical ailments, stress, emotional disorders and occupational risks associated with daily activity.
\end{abstract}

Key words: Active pauses; job performance; dysergonomic conditions

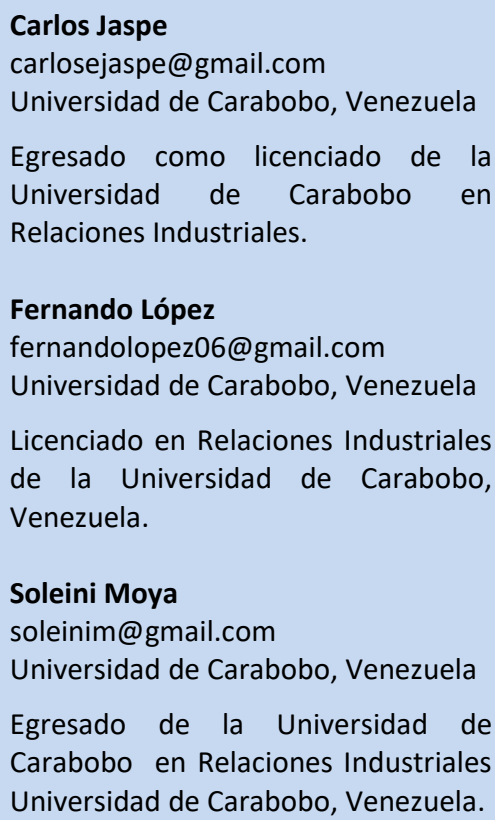




\section{INTRODUCCIÓN}

El ámbito laboral, considerando lo planteado por Robles (2012), a nivel mundial ha sido reconocido como un espacio adecuado para la promoción de la salud y posteriormente fue ratificado por la Organización Mundial de la Salud (OMS) y la Organización Internacional del Trabajo (OIT). La denominada pausa activa laboral tiene su origen en Polonia en el año 1925. Su primera denominación fue gimnasia de pausa y era destinada a los operarios de fábricas. Años más tarde surgió en Holanda y Rusia, y a comienzos de los años 60 se trasladó a Bulgaria, Alemania, Suecia y Bélgica. Durante la década de los 60 , en Japón hubo una consolidación y obligatoriedad de la pausa activa laboral.

Desde hace 15 años, cuando en las grandes compañías descubrieron que sus trabajadores se quejaban más de enfermedades relacionadas con el trabajo, los profesionales de la salud ocupacional introdujeron el termino de pausas activas entre su personal de trabajo para disminuir el número de casos de trabajadores por distintas enfermedades en relación al trabajo a su vez aumentar el desempeño laboral.

Con relación al desempeño laboral según Chiavenato (2009) lo define como las acciones o comportamientos observados en los empleados que son relevantes al logro de los objetivos de la organización. En efecto, afirma que un buen desempeño laboral es la fortaleza más notable con la que cuenta una organización.

En muchos países se ha tomado muy en cuenta las pausas activas laborales como un mecanismo para mejorar el desempeño laboral y los trabajadores se sientan más satisfechos y que pueden canalizar todo su potencial hacia actividades productivas, reduciendo enfermedades profesionales y con esto el ausentismo laboral que es perjudicial para cualquier organización. Por ello, múltiples organizaciones alrededor del mundo fomentan esta práctica y cada vez son más personas que quieren rendir en sus trabajos, ser más competitivos pero claro sin descuidar su salud mental y física.

Básicamente, las pausas activas comprenden una serie de movimientos iniciales de las articulaciones (que además de proteger las articulaciones, reducen la tensión de los músculos y tendones), seguidos de estiramientos y ejercicios específicos para distintos grupos musculares que incluyen el cuello, las manos, las extremidades superiores e inferiores y la espalda, acompañados de respiraciones rítmicas, profundas y lenta. Lo que conlleva a que las personas recuperen energías para un desempeño eficiente en su trabajo, a través de diferentes técnicas y ejercicios que ayudan a reducir la fatiga laboral, trastornos osteomusculares y prevenir el estrés. Además, contribuyen a disminuir la fatiga física y mental y a integrar los diferentes grupos de trabajo durante la ejecución de las pausas.

Según Castro, y otros (2011) afirman que es deseable que se realicen pausas adecuadas preferiblemente flexibles que produzcan cambio en la posición y mejoramiento en el proceso de los grupos musculares afectados por la actividad. Adicionalmente, mencionan que, acompañadas de la higiene postural en el puesto de trabajo, buenos hábitos nutricionales, de sueño y del tiempo libre, las pausas activas, pueden prevenir diferentes dolencias y enfermedades ocupacionales.

En tal sentido la Norma COVENIN 2742 (1998) que rige en Venezuela, indica que tan solo 15 minutos por cada dos horas continuas de trabajo, previene la fatiga 
muscular, por lo cual mitiga los factores para la aparición de lesiones músculoesqueléticas, aumenta la productividad, permite mayor capacidad de concentración, modifica el nivel energético del individuo, ya que la respiración es fundamental; y desarrolla la implementación de hábitos de vida saludables.

Así pues, la implementación de las pausas activas busca valorar la salud física y mental de sus trabajadores, como también mejorar su desempeño laboral, más allá de cumplir con una ley o con un reglamento impuesto, debería estar escrito en las políticas internas de cada empresa y en su oportuna aplicación.

El trabajador que está frente al computador durante 7 horas continuas aproximadamente, sufre serias molestias visuales, problemas en su columna, espalda, manos, piernas, cabeza, etc., aumentando el cansancio a medida que el tiempo transcurre, este se vuelve permanente y no permite el desenvolvimiento total de la persona, por lo cual el hacer una pausa durante la jornada es una manera efectiva de recuperar energías y oxigenar la mente.

La implementación de las pausas activas como estrategia preventiva de la fatiga y el mal desempeño laboral por condiciones disergonómicas de una empresa manufacturera ubicada en el municipio Valencia, Estado. Carabobo, permitirá dar respuesta a las siguientes interrogantes: ¿Qué beneficios obtendrán los trabajadores por medio de la implementación de las pausas activas? ¿Cuál es el nivel de desempeño laboral de los trabajadores? ¿Cómo reconocer los factores que generan los riesgos disergonómicos en el puesto de trabajo? ¿Cómo impactan las pausas activas en las condiciones físicas de los trabajadores?
Asimismo, se planteó como Objetivo General el analizar la aplicación de pausas activas como estrategia preventiva de la fatiga y el mal desempeño laboral por condiciones disergonómicas en actividades administrativas de una empresa manufacturera, ubicada en el municipio Valencia, Estado Carabobo. Para ello se identificó la presencia de condiciones disergonómicas en actividades administrativas de una empresa manufacturera en Valencia, Estado Carabobo, y se caracterizó la fatiga física y mental en actividades administrativas de los trabajadores, evaluando el impacto de las pausas activas en el desempeño laboral de los trabajadores del área administrativa de una empresa manufacturera en Valencia Estado Carabobo.

En el marco de estas líneas introductorias del estudio, es pertinente resaltar que de acuerdo a González (2013), las pausas activas o gimnasia laboral como una serie de ejercicios físicos y estiramientos de los diversos grupos musculares, implicando movimientos articulares que realizan los trabajadores de todos los segmentos del cuerpo humano. Se pueden incluir ejercicios de respiración, fortalecimiento y postura, se recomienda que sean realizados en un espacio acogedor y saludable.

Las pausas activas hacen parte de programas de salud ocupacional, los cuales buscan el bienestar del empleado en su sitio de trabajo; por lo tanto es vital que esto se realice en las organizaciones puesto que esto garantizará la seguridad en el sitio de trabajo o posibles enfermedades profesionales.

Según González (2013) las pausas activas son ejercicios frecuentemente realizados de forma grupal. Posteriormente es recomendable se eduque y enseñe a los 
trabajadores como deben realizar las pausas activas para que ellos puedan realizar los ejercicios de forma autónoma. Se debe considerar que estas pausas no interfieran con los tiempos de producción empresarial y es necesario tomar en cuenta las características específicas de cada área de trabajo.

Las pausas activas están destinadas a disminuir el esfuerzo diario en el área de trabajo y predestinado a la educación, formación y recuperación de rutinas diarias para mejorar la calidad de vida de los trabajadores.

Según Castro y otros (2011) las pausas activas aumentan la armonía laboral a través del ejercicio físico y la relajación, alivian las tensiones laborales producidas por malas posturas y rutina generada por el trabajo, incrementan el rendimiento o desempeño laboral en la ejecución de las labores, ya que disminuyen el estrés laboral, los factores generadores de trastornos músculoesqueléticos de origen profesional que repercuten principalmente en cuello $y$ extremidades superiores, las ausencias al trabajo.

Vélez (2011) señala en cuanto al tiempo y lugares de las pausas activas lo siguiente, que se deben realizar en cualquier momento del día cuando se sienta pesadez corporal, fatiga muscular, incomodidad, angustia o sobreexcitación síquica; también pueden establecerse pausas rutinarias en mitad de la jornada laboral (una vez en la mañana y una en la tarde). Para su ejecución se debe estar cómodo y reposado, aflojar la ropa y sentir la acción relajante sobre cada parte del cuerpo que entra en acción.

Según Bonilla (2012) el sedentarismo entendido como la falta de movilidad o inactividad mínima necesaria que requiere el organismo para mantenerse saludable, es asumido como parte de la vida diaria y con él sus múltiples consecuencias como las enfermedades cardiovasculares y el aumento de la morbilidad por enfermedades asociadas como la obesidad, el estrés y el consumo de alcohol. Son muchos los factores que influyen o conllevan a momentos que incapacitan, junto con el estrés se encuentra otra situación que además de coadyuvar a generar molestias físicas es una de las situaciones que más está enfermando a la población especialmente la juvenil y la laboralmente activa.

El sedentarismo lo define la Real Academia de la Lengua Española (2011) como: "Dicho de un oficio o de un modo de vida de poca agitación o movimiento", siendo así, es claro que define los estilos de vida a los que la sociedad actual ha llegado, según estudios realizados se encuentra que el sedentarismo afecta significativamente el sistema músculo esquelético, además desencadenando otras patologías propias del sistema locomotor como son la osteopenia y la osteoporosis, la poca movilidad afecta también el sistema tendinoso por falta de estímulo o de tensión.

Se puede también establecer que afecta el sistema cardiovascular provocando hipertensión arterial, infarto del miocardio y arritmias, es por esto que la Fundación española de cardiología y la Fundación española del corazón se unen para promover la realización de actividades físicas regularmente. En este caso ellos plantean los riesgos a que están expuestas las personas sedentarias ya que se puede sufrir de un ataque cardiaco.

Pero, además, estimulan la práctica del ejercicio teniendo en cuenta que la actividad física continuada tiene muchos efectos beneficiosos para la salud como la pérdida de peso, ayuda a dejar de fumar, reduce el 
estrés, disminuye la presión arterial, ayuda a controlar los niveles de azúcar en la sangre y eleva los niveles de colesterol HDL (colesterol bueno). Siendo la actividad física como un estilo de vida que modifica aspectos conductuales de las personas, también es cierto que aumenta el autoestima impactando directamente en el estado de ánimo; al terminar cada día la jornada laboral el cuerpo se siente agotado y es allí cuando se necesita de la actividad física para restaurar los sistemas musculares y tendinosos logrando la energía y vitalidad que el cuerpo está necesitando y que el ejercicio proporciona.

Por otro lado, Salas (2012) define el desempeño laboral como el comportamiento o la conducta real de los trabajadores, tanto en el orden profesional y técnico, como en las relaciones interpersonales que se crean en la atención del proceso salud / enfermedad de la población, el cual influye a su vez, de manera importante el componente ambiental. Por tanto, existe una correlación directa entre los factores que caracterizan el desempeño profesional y los que determinan la calidad total de los servicios de salud.

Según Chiavenato (2009) la evaluación del desempeño es una valoración, sistemática, de la actuación de cada persona en función de las actividades que desempeña, las metas y los resultados que debe alcanzar, las competencias que ofrece y su potencial desarrollo. Este autor nos explica que es un proceso que sirve para juzgar o estimar el valor, la excelencia y las competencias de una persona, pero, sobre todo la aportación que hace al negocio de la organización.

Con relación a la ergonomía, Barquín (2000) indica que la ergonomía es una ciencia multidisciplinaria, cuyos principios están basados en las capacidades físicas y psíquicas de los trabajadores para diseñar los puestos de trabajo y equipos de empresa, cuya meta es optimizar la relación entre el trabajador y sus actividades laborales. Lo antes mencionado, permite inferir que la ergonomía incorpora una serie de soluciones destinadas a mejorar las condiciones de trabajo a fin de eliminar o reducir la presencia de fatiga o alteraciones producidas por sobrecarga física, disminuir las bajas laborales o el ausentismo y contribuir a aumentar la satisfacción y el rendimiento en el lugar de trabajo.

Se entiende como Condición Disergonómica, condiciones, herramientas u equipos que pueden ocasionar un perjuicio a la salud del hombre en el trabajo, ergonómicamente hablando. Es lo equivalente a decir "no ergonómico". Son todas aquellas condiciones, posiciones $y$ circunstancias como se realiza un trabajo, que pueden producir la inadecuada adaptación de los medios del trabajo al trabajador o viceversa, los cuales son capaces de originar una lesión o daño a la salud.

Cabrera (2011) señala que la Condición Disergonómica es una condición de trabajo que presenta factores de riesgos disergonómico, lo que quiere decir el autor es que son todas aquellas características generales del trabajo o el medio ambiente de trabajo que puedan resultar en daño físico para el trabajador.

Según Vidal (2010) la fatiga física y mental es la disminución de la capacidad física y mental de un individuo, después de haber realizado un trabajo durante un periodo de tiempo determinado. La fatiga física se utiliza para definir una serie de situaciones cuyo resultado final supone una disminución de la capacidad de trabajo y de 
la resistencia del organismo, mientras que la fatiga mental se produce una disminución de los niveles de atención, provoca una disminución significativa de la motivación hacia el trabajo.

\section{MATERIALES Y MÉTODO}

La investigación fue de campo, ya que los datos se extrajeron en forma directa de la realidad, a través del uso de instrumentos para recolectar la información. La investigación fue también descriptiva, porque se tomaron las referencias que aportaron información vital e importante para la investigación, y así se lograron los objetivos planteados. Méndez (2003), con respecto a la investigación descriptiva expresa que, de acuerdo a Rodríguez y otros (2012), “las estrategias metodológicas, corresponden a la sección donde indican y describen las etapas y herramientas de planificación y control en la ejecución de la investigación que se desarrollará" ( $\mathrm{s} / \mathrm{n})$, es decir las estrategias metodológicas deben ir de acuerdo al diseño de investigación. Además las estrategias metodológicas pueden ser representadas por medio de un cuadro metodológico. Para Delgado de Smith (2008), el cuadro metodológico se describe como una buena herramienta de apoyo que contiene todos los objetivos específicos. Es una entre muchas vías para ordenar, clasificar y ejecutar la investigación.

Por tal motivo, los resultados que se obtuvieron también se lograron por el seguimiento de lo que ha sido elaborado y sistematizado por autores que ya trabajaron en el asunto anteriormente. Igualmente, este trabajo estuvo basado en una percepción de las variables metodológicas utilizadas en el estudio, la población total seleccionada para el desarrollo de este estudio, fue un total de (21) trabajadores pertenecientes al área administrativa de la empresa en estudio, este estudio tiene una población finita o muy reducida lo cual conviene tomarla en su totalidad, lo que metodológicamente se conoce como muestra no probabilística intencional. Por tanto, la muestra de estudio estuvo integrada por veintiún (21) sujetos.

Las técnicas e instrumentos de recolección de información, por ser una investigación descriptiva en el ámbito social, fueron las técnicas de observación directa, la encuesta y revisión de documentos.

En este caso, estas técnicas se utilizaron en la muestra correspondiente al personal administrativo a los efectos de conocer el impacto de las pausas activas en el desempeño laboral de los trabajadores, se utilizó como instrumento un cuestionario estructurado, y para la técnica de observación se utilizó como instrumento una guía de observación.

La validez del instrumento se realizó a través de juicios de expertos, los mismos, fueron seleccionados de manera independiente, los cuales determinaron la relevancia y congruencia de las preguntas con el contenido teórico, la claridad en la redacción y el sesgo en la formulación de los ítems, es decir, si sugieren o no una respuesta.

Luego de aplicar los instrumentos diseñados para la recolección de información se procedió a manejar los datos y ofrecer el producto de este análisis, lo cual se realizó por medio de la tabulación de resultados en una tabla de frecuencias y porcentajes para luego aplicar una herramienta de representación gráfica. En este caso, se utilizaron las gráficas de barra donde se pueden observar los resultados que se obtuvieron del cuestionario con su debido valor y porcentaje. 
Dado el límite de páginas establecidos para este tipo de artículo, a continuación resumiremos lo más importante de las respuestas dadas a por los encuestados, a fin de que estas sean de alguna manera referentes para futuros estudios en esta materia, para ello se ha elaborado el siguiente cuadro.

\section{Movimientos físicos con frecuencia dentro de las funciones laborales}

En las observaciones realizadas los investigadores constataron que el personal del área administrativa, requieren en su mayor parte del tiempo la realización de esfuerzos físicos en su estación de trabajo, sin embargo es importante destacar que si no realizan pausas activas puede verse afectado las condiciones físicas y el rendimiento laboral de los trabajadores.

\section{Actividades que son ejecutadas en los puestos de trabajo}

Se puede decir, que la mayoría de las personas encuestadas manifestaron que sus actividades diarias no requieren de un mayor esfuerzo físico, sin embargo, se pudo observar que en las diferentes estaciones de trabajo si deben realizar esfuerzos físicos diariamente que requieren movilidad de su puesto de trabajo a otros sitios dentro de la misma área ya que son parte de su rutina de trabajo.

\section{El diseño del puesto de trabajo y el tiempo de estancia}

No hubo uniformidad en las respuestas que permitan negar o afirmar lo preguntado. Es importante destacar el deber de las organizaciones de garantizar condiciones seguras y saludables para los trabajadores, sin embargo se pudo observar que cuando las jornadas de trabajo se prolongan, gran parte del personal administrativo tienden a mantener posturas inadecuadas a nivel de la espalda, la cual trae consecuencias en la zona del tronco y disminuye de esta manera el rendimiento laboral y prolonga a su vez el cansancio físico y mental.

\section{Postura apropiada en la función laboral}

El 33\% de la población está totalmente de acuerdo que al momento de realizar las actividades en su estación de trabajo mantienen una postura adecuada en su espalda, el $43 \%$ está de acuerdo, por su parte un $10 \%$ manifiesta estar ni de acuerdo ni en desacuerdo, mientras que el $14 \%$ restante de la población encuestada está en desacuerdo. Adoptar y mantener una buena postura es fundamental para la salud de la espalda y todo el cuerpo en general, se observó que gran parte del personal administrativo mantiene una postura adecuada, sin embargo varias personas de edades comprendidas entre 30 y 50 años, realizan trabajos frente a las computadoras, sentadas durante horas sin alternar la postura y bajo condiciones disergonómicas, y puede generar problemas musculo esqueléticos para los trabajadores expuestos.

\section{Comodidades al momento de cumplir la función laboral}

El $10 \%$ de la muestra indicaron estar totalmente de acuerdo en que el trabajo que realizan les exige mantener en el cuello una postura incomoda por tiempo prolongado, el $14 \%$ manifiesta en estar ni de acuerdo ni en desacuerdo, mientras que la población restante están de acuerdo con un $29 \%$ y totalmente de acuerdo con un38\%. La mayor parte de las respuestas al ítem 5, se 
evidenció que existe un índice bajo hasta indeciso de que las personas presentan posturas incómodas por tiempo prolongado, ya sea tener una mala postura o como permanecer mucho tiempo en una misma posición todos los días puede ocasionar sobrecarga y tensiones en varias parte del cuerpo que pueden finalizar en lesiones principalmente a nivel lumbar y del cuello.

\section{Tiempo de descanso en la jornada laboral}

Un 48\% está de acuerdo, que durante la jornada laboral cuentan con pausas suficientes para descansar, $33 \%$ está totalmente de acuerdo, así mismo un 10\% está en desacuerdo, un $5 \%$ está totalmente en desacuerdo y $5 \%$ ni de acuerdo ni en desacuerdo.

Con lo anteriormente descrito, los trabajadores en su mayoría aseguran que se encuentran de acuerdo, que cuentan con pausas suficientes para descansar, sin embargo durante la investigación se pudo observar la ausencia de esta práctica y del trabajo acumulado que puede afectar su desempeño laboral.

\section{La energización forma parte en mi horario de trabajo}

Un $48 \%$ está de acuerdo que en su trabajo puede tomar un tiempo para recuperar energía, un 38\% está totalmente de acuerdo, mientras que un 5 por ciento está ni de acuerdo y ni en desacuerdo, así mismo un 5 por ciento está en desacuerdo y otro 5 por ciento está totalmente en desacuerdo. En virtud a la descripción anterior, se puede apreciar que los trabajadores en su gran mayoría afirman que pueden tomarse un tiempo para recuperar energías, sin embargo se observó que usualmente ese tiempo no es tomado ya que la carga de trabajo suele ser mayor sobre todo en el departamento de finanzas.

\section{El cansancio afecta el desempeño laboral}

Un 38\% está totalmente de acuerdo que el cansancio afecta su desempeño laboral, mientras que otro $38 \%$ está de acuerdo, un $10 \%$ ni de acuerdo ni en desacuerdo, un $10 \%$ en desacuerdo y el 5 por ciento manifiesta estar totalmente en desacuerdo. Se puede determinar que un significativo número de encuestados se encuentra totalmente de acuerdo y de acuerdo con respecto al cansancio que producen sus labores y su desempeño. Es importante resaltar que las personas que se encuentran cansadas tienen mayor dificultad para lograr la eficacia en las tareas asignadas lo que afecta directamente la eficiencia en el trabajo y no podrán cumplir con todas las actividades del puesto de trabajo en los tiempos requeridos, al encontrarse en esta situación no le permite analizar y realizar las actividades de forma eficiente, provocando que la organización no obtenga la información precisa y perdiendo recursos.

\section{La postura física puesto de trabajo}

El $24 \%$ de las personas encuestas indican que la postura que adoptan en el puesto de trabajo le genera molestia, mientras que el $19 \%$ se mantienen estáticos y ni están de acuerdo ni en desacuerdo y el $24 \%$ de la población restante manifestaron en estar en desacuerdo y totalmente en desacuerdo. Se pudo observar que las personas que hacen vida en los distintos departamentos administrativos con frecuencia mantienen posturas estáticas durante la realización de sus actividades, esto puede estar motivado a 
la falta de conocimiento sobre educación postural especialmente en los trabajos administrativos donde se observa la presencia de sedestación prolongada, generando estas condiciones riesgos para la salud de los trabajadores, las cuales con el pasar del tiempo resultan perjudiciales porque afectan su salud.

\section{Molestias físicas en la función laboral}

El 14\% de las personas encuestas están totalmente de acuerdo y manifiestan que al final de sus labores de trabajo sienten molestia a nivel del cuello, el $29 \%$ coinciden en estar de acuerdo, mientras que un $10 \%$ ni está de acuerdo ni en desacuerdo, y gran parte de la muestra está en desacuerdo con un $29 \%$ y totalmente en desacuerdo con un 19\%. Durante la aplicación del cuestionario a final de la tarde, se pudo observar que un grupo de personas de edades comprendidas entre 35 y 60 años, si presentan molestias en la zona del cuello, mientras que otra parte de la población de menor edad, se encontraban físicamente en mejores condiciones. Es importante destacar que la mala postura, ya sea sentado o de pie también genera molestias a nivel de miembros superiores.

\section{Las pausas en el trabajo permiten reducir la tensión muscular}

Un 33\% aseguró estar totalmente de acuerdo en que las pausas activas permiten reducir la tensión a nivel muscular durante la jornada laboral, un 57\% opina estar de acuerdo con esta premisa representando la mayoría de la población, un $5 \%$ ni acuerdo ni desacuerdo y $5 \%$ totalmente desacuerdo. Por ende la mayoría de las personas encuestadas están de acuerdo con que la realización de las pausas activas reflejan en ellos una reducción de la tensión muscular generadas por sus actividades diarias, sin embargo aún hay parte de los trabajadores en total desacuerdo con esta premisa, manifestando así la necesidad de seguir reforzando mediante la teoría y práctica los beneficios reales de las pausas activas.

\section{Beneficios de tomar un buen descanso}

Un 62\% de los trabajadores indican según su percepción estar totalmente de acuerdo que la toma de un breve descanso durante sus actividades diarias ayuda a prevenir las lesiones osteomusculares y un $38 \%$ considera estar de acuerdo. Las lesiones osteomusculares son un conjunto de lesiones inflamatorias o degenerativa de los músculos, tendones, articulaciones, ligamentos y nervios, que generalmente se localizan en la zona del cuello, espalda, hombros, codos, muñeca y manos. De allí se deriva que la mayoría de los trabajadores estén conscientes de lo importante que es tomar durante la jornada laboral un breve descanso a fin de evitar daños en la salud física.

\section{Las pausas activas ayudan a corregir las posturas disergonómicas en el trabajo}

Según la población encuestada un 52\% responde estar totalmente de acuerdo que las pausas activas ayudan a corregir las posturas disergonómicas en el trabajo, un $43 \%$ afirma estar de acuerdo y solo un $5 \%$ ni de acuerdo, ni en desacuerdo. El alto porcentaje de aceptación a esta interrogante puede estar relacionada con el conocimiento que poseen los trabajadores sobre los beneficios de las pausas activas, esto se debe a que las pausas activas son de gran importancia ya que al realizarlas se obtienen beneficios que previenen dolores a nivel 
cervical y lumbar, beneficiando de igual forma el sistema circulatorio y óseo, mejorando el desempeño laboral.

\section{Descanso en la rutina de trabajo}

De la totalidad de encuestados un $57 \%$ afirma estar de acuerdo que un breve descanso en la rutina de trabajo ayuda a disminuir el estrés y la fatiga mental, un $38 \%$ de acuerdo con esta pregunta y un $5 \%$ indica que ni de acuerdo, ni desacuerdo. Las pausas activas a pesar de ser de un lapso pequeño de ejecución permiten desconectar al trabajador de presiones que generan las actividades diarias y así este breve descanso estimula al cuerpo a reponer las energías tanto físicas como mentales, es por esto que la mayoría de la población encuestada asegura estar totalmente de acuerdo con esta premisa.

\section{Manejos de las emociones en la función laboral}

De acuerdo a la población encuestada un $43 \%$ responde estar totalmente de acuerdo y de acuerdo respectivamente a lo que sugiere esta interrogante, un $10 \%$ opina ni de acuerdo, ni desacuerdo y $5 \%$ en desacuerdo.

La mayoría de los trabajadores afirma positivamente que al realizar las pausas activas, son unos de los beneficios que brinda y suelen ser importantes dentro de una organización, ya que contribuye a que los trabajadores mantengan buenas relaciones entre ellos.

\section{Concentración dentro de las actividades o funciones laborales}

Un $62 \%$ el cual representa la mayoría responde estar totalmente de acuerdo que tomar un breve descanso ayuda a poner más atención y concentración en las labores, un
$33 \%$ opina estar de acuerdo y un $5 \%$ en desacuerdo.

Todo trabajador en su jornada laboral está expuesto a un conjunto de requerimientos psicofísicos que hacen parte de la carga de trabajo, a la que se encuentra sometido a lo largo de su vida profesional, tareas repetitivas, sobreesfuerzos, posturas corporales mantenidas, forzadas, la toma de decisiones, la gestión organizacional y el trabajo monótono son factores que pueden afectar el normal funcionamiento del cuerpo y de la mente en el trabajo, por lo anterior es fundamental realizar pausas activas que le permitan al trabajador continuar con sus labores con toda la concentración y disposición.

\section{Las pausas activas permiten reflexionar} los procesos de trabajo y desarrollar hábitos que mejoran el desempeño

De la totalidad de encuestados un $38 \%$ indica estar totalmente de acuerdo, también un $57 \%$ que representa la mayoría de la población responde estar de acuerdo en que las pausas activas permiten repensar los procesos de trabajos y desarrollar hábitos que mejoran el desempeño laboral, sin embargo un $5 \%$ expresa que está ni acuerdo, ni en desacuerdo. Debido a que las pausas activas brindan al trabajador lograr mayor concentración luego de realizarlas, permiten a su vez que el mismo adecue su forma de trabajo y visualice cuales son las causas que lo llevan a caer en la rutina, monotonía y otros factores de la jornada laboral que le impiden cumplir con sus actividades, para así fomentar un hábito de manera voluntaria de aplicar las pausas activas de tal forma que impacten significativamente en su desempeño laboral y lograr sus objetivos individuales. 
Mejoraras en la productividad del desempeño laboral acompañado del descanso

Del total de encuestados un $57 \%$ asegura estar totalmente de acuerdo y un $43 \%$ dice estar de acuerdo con que se puede mejorar la productividad de su desempeño cuando hacen pausas activas en su jornada. Las pausas activas luego de realizarse permiten al trabajador reconectarse $y$ enfocarse en cuáles son sus objetivos primordiales en el puesto de trabajo que desempeña en la organización para así tener un mayor porcentaje de efectividad en cada una de sus actividades, es por esto que muchas empresas en la actualidad se apoyan en esta herramienta para inculcarle a sus trabajadores los beneficios que trae consigo la aplicación de las mismas tanto a nivel organizacional como personal.

\section{Objetivos de trabajo en el tiempo esperado}

El $67 \%$ están totalmente de acuerdo que cumplen con los objetivos en el tiempo que se le es asignado y un 33\% están de acuerdo. Respecto a estos resultados, se observa que el $100 \%$ de los encuestados manifiesta dar cumplimiento a los objetivos en el tiempo asignado, sin embargo es posible inferir que al estar enfocados en el cumplimento de sus actividades, incrementa así la posibilidad de aparición de factores de riesgo que afecten el desempeño laboral de los trabajadores.

De acuerdo al estudio realizado, con relación a los trabajadores y la actividad se interpretó que las pausas activas en el desempeño laboral, interrumpen la postura que le genera esa fatiga y puede verse una mejora en el desempeño laboral.

Se precisó mediante la investigación realizada en la empresa, que al no realizar pausas activas incide notablemente en el rendimiento de los trabajadores, por lo cual se recomienda crear planes de acción que mejoren la productividad de los trabajadores. El hecho de no tener planes de trabajo, no realizar pausas activas y que existan condiciones disergonómicas afectan al trabajador y a su rendimiento.

Ciertamente las pausas activas influyen en el desempeño laboral de los trabajadores porque se identificó la presencia de condiciones disergonómicas como las posturas forzadas o mantenidas durante mucho tiempo, la repetitividad en esas posturas, sentados por mucho tiempo frente al computador y no interrumpen su jornada generando fatiga y cansancio a los trabajadores y eso a su vez genera bajo desempeño laboral.

\section{CONCLUSIONES}

Se presentó en el Departamento de finanzas un desinterés para realizar pausas activas, ya que se encontraban muy ocupados con demasiada carga de trabajo, siendo esta actitud recurrente en ese departamento, el resto del personal fue más receptivo, sin embargo no realizan dichas pausas en su jornada laboral dejando esto por sentado en sus opiniones y comentarios luego de realizarles una actividad de pausas activas.

La receptividad de los trabajadores ante la pregunta ¿Siento que un breve descanso en la rutina de trabajo me ayuda a disminuir el estrés y la fatiga mental?, genera una ventaja para la aplicación de un programa de pausas activas que se derive en beneficios de todos los actores laborales.

El compromiso emocional de un trabajador con su empresa es mucho más importante que cualquier valor corporativo 
que se pueda transmitir. Las repercusiones de tomarse una pausa activa en el trabajo pueden incluso llegar a disminuir las ausencias laborales por lo que se convierte en un ambiente laboral ameno para el trabajador, es por esto que la mayoría de los encuestados afirman estar totalmente de acuerdo que las pausas activas contribuyen a mejorar la autoestima y las relaciones entre compañeros.

\section{REFERENCIAS}

Barquín (2000) Ergonomía. Recuperado de http://mriuc.bc.uc.edu.ve/bitstream/ha ndle/123456789/2572/Lrossodivita.pdf? seque

Bonilla F. (2012). El sedentarismo. Andalucía: Edición Delfín Galiano

Cabrera, L. (2011) Condición Disergonómica. Recuperado de:http://mriuc.bc.uc.edu.ve/bitstream/ handle/123456789/2583/\%C3\%81hern \%C3\%A1ndez.pdf?sequence=1. Consulta 2018, Abril 05

Castro, C., y Munera, V. (2011) Beneficios de las pausas activas. Bogotá, Grupo Editorial Norma

Chiavenato, (2009). Definición de Evaluación de desempeño laboral. Recuperado de http://www.monografias.com/trabajos1 01/consideraciones-acerca-evaluaciondel-desempenolaboral/consideraciones-acercaevaluacion-del-desempeno-laboral. html

González, P. (2013). Pausas activas en el trabajo: ejercicios físicos y mentales que energizan. Chile: Universidad Mayor Sochergo

Norma Venezolana COVENIN 2742, (1998). Condiciones Ergonómicas en los puestos de trabajo en terminales con pantalla catódicas de Datos. Aprobada en reunión $\mathrm{N}^{\circ} 149$. Noviembre 12,1997

Organización Mundial de la Salud (2018). Inactividad física: un problema de salud pública mundial. Recuperado de http://www.who.int/dietphysicalactivity/fac tsheet inactivity/es/

Real Academia Española (2011). Definición de sedentarismo. Recuperado de http://dle.rae.es/?w=diccionario\&origen=RE DLE. Consulta: 2018, Marzo 25

Robles, L. (2012). Origen de las pausas activas. Recuperado de https://es.slideshare.net/julietayeya/pausas -activas-14973498

Vidal, J. (2010). Fatiga física y Mental. Recuperado de www.uv.es/sfpenlinia/cas/323fatiga_mental .html.

Vélez, H. (2011). Tiempo y lugares en los que se deben realizar las Pausas Activas Laborales. Dificultades para implementar las pausas activas. Universidad de las Américas. Recuperado de https://dspace.ups.edu.ec/bitstream/12345 6789/7771/1/UPS-CT004632.pdff 